\title{
A Complex Network-Based Approach for Analysis of Drug Usage
}

\author{
Lianhong Ding ${ }^{1, *}$, Ke Xuan², Ruiping Yuan ${ }^{1}$ \\ ${ }^{1}$ School of Information, Beijing Wuzi University, Beijing, China \\ ${ }^{2}$ Jinan Second People's Hospital, Jinan, China
}

Email address:

lhdingbwu@sina.com (Lianhong Ding)

${ }^{*}$ Corresponding author

\section{To cite this article:}

Lianhong Ding, Ke Xuan, Ruiping Yuan. A Complex Network-Based Approach for Analysis of Drug Usage. Advances in Networks. Vol. 4, No. 1, 2016, pp. 6-12. doi: 10.11648/j.net.20160401.12

Received: October 31, 2016; Accepted: November 14, 2016; Published: November 25, 2016

\begin{abstract}
The status of drug usage for patients is important for the research about clinical application. This paper analyzes the drug usage in an eye hospital. Two approaches are adopted for the analysis procedure. There are statistics and complex theory. Four networks for different kinds of drugs are built first. Then degree property of these networks is used. Finally, the comparison between the two methods is conducted. The comparison results show node degree of drug network can reflect the drug using frequency well.
\end{abstract}

Keywords: Drug Usage, Complex Network, Degree, Eye Hospital

\section{Introduction}

The study of complex networks is a young and active area of scientific research [1]. Recent years, studies about the properties of networked systems, such as the power grid, transport network and scientific collaboration network, increased apparently [2-4]. Related research, to a large extent, focus on properties such as the so called "small-world effect" and skewed degree distributions [5]. Here, we highlight the degree property and its application in the analysis of drug usage for outpatients in an eye hospital.

Vogler focused on whether socioeconomic determinants influence the use of prescribed and non-prescribed medicines in eight Central and Eastern European countries. The analysis showed the pharmaceutical systems in the eight countries were, to varying degrees, characterized by a lack of (public) funding, thus resulting in high and growing shares of private financing, inefficiencies in the selection of medicines into reimbursement and limitations in medicines availability [6]. Adnan et al. introduced the traditional use of plants in the war affected region of northwest Pakistan. Of the 107 species of ethnomedicinal plants reported, the most common use is for carminative purposes, the next most common use is for blood purification [7]. Huang et al. studied the traditional Chinese medicine usage in children in Taiwan through statistical method. It was found that herbal remedies were the most commonly used therapeutic approach, then manipulative therapy and acupuncture [8]. Yen et al analyzed the characteristics of traditional Chinese medicine use for children with allergic rhinitis [9].

In China, chemical medicines, herbal medicines and Chinese patent medicines are widely used at the same time. The use analysis of drugs is carried out for these three kinds of medicines respectively. The analysis is carried out first by statistics method then through the complex network theory. At last the analysis results through these two methods are compared.

Section 2 of this paper discusses the method to collect and prepare the data set for the analysis. Section 3 is the drug use analysis by statistics method. Section 4 is the drug use analysis through complex network theory. The analysis results through these two methods are compared and discussed in section 5 .

\section{Data Collection and Data Processing}

The basis of the analysis is a data set about the prescription drugs form an eye hospital of Jinan City in China, named as Jinan $2^{\text {nd }}$ people's hospital. Jinan $2^{\text {nd }}$ people's hospital is equipped with internal, external, women, traditional Chinese 
medicine, oral and other departments. Ophthalmology department is the most important department for this hospital.

The data set comes from the history data in the clinical treatment. 65535 records about the prescription drugs used by outpatients from September 14, 2013 to January 2, 2014 constitute the final data set for our analysis. Initially there are some duplicate records. When the duplicate records are deleted there are 64186 records remained in our data set.

At first, there are 808 kinds of drugs in our data set. During the procedure of data processing, we found that a same kind of drug may be recorded as two or more kinds of drugs because of different manufacturers, different dosage forms or different specifications in the data set. For example, there are seven kinds of $0.9 \%$ sodium chloride injection and eight kinds of $5 \%$ glucose injection. This kind of phenomenon will influence the accuracy of the analysis result. So the main work for the data processing is to unify and merge the kinds of drugs. After this step, there are 583 kinds of drugs in the data set finally.

Different from western countries, except for western medicine, traditional Chinese medicine (TCM) is widely used in China also [10]. TCM is widely used in China and is becoming increasingly prevalent in Europe and North America. So chemical medicine and herbal medicine are prevalent both. Herbalism (also named as herbology or herbal medicine) is the use of plants for medicinal purposes, and the study of botany for such use [11]. Herbal drugs are produced according to good manufacturing practice (GMP) and utilized in accordance with good clinical practice (GCP). Chinese medicine diagnostics is accomplished through the four clinics of the look and smell. It is the responsibility for a doctor to write specific Chinese medical formulae as prescription for each patient. Usually, all the medicinal materials on the prescription are decocted to form one decoction by patients themselves. In 2001, a total of 1249 traditional Chinese medicines were listed in China's national essential drugs list [12].

With the development of modern TCM, Chinese patent medicine is used extensively in recent years. Each kind of Chinese patent medicine is produced by more than one kind of medicinal materials. The common forms of Chinese patent medicine may be pill, tablet or oral liquid. The development of Chinese patent medicine frees the patient from medicine-decocting. So there are three categories of drugs in our data set. They are chemical medicine, herbal medicine and Chinese patent medicine. There are 269 kinds of chemical medicines, 243 kinds of herbal medicines and 71 kinds of Chinese patent medicines in our data set.

\section{Drug Use Analysis by Statistics}

From September 14, 2013 to January 2, 2014, chemical drugs are used 47913 times, herbal medicines are used 7069 times and Chinese patent medicines are used 9204 times in Jinan $2^{\text {nd }}$ people's hospital. The average value of using frequency for all these 583 kinds of drugs is 109.91, the average using frequency for chemical medicine is 178.12 , the average using frequency of herbal medicine is 29.09 and the average using frequency for Chinese patent medicine is 129.63 .

Table 1 lists the drugs which are used most frequently in the clinical diagnosis for outpatients. The top 10 drugs are Pranoprofen Eye Drops, Dixin Eye Drops, 0.9\% Sodium Chloride Injection, Compound Tropicamide Eye Drops, Lubricant Eye Drops, Sodium Hyaluronate Eye Drops, Mecobalamin Capsules, Bendazac Lysine Eye Drops, Esculin and Digitalisglycosides Eye Drops and Polyvinyl Alcohol Eye Drops respectively. They are all chemical medicines and eight of them are eye drops.

It should be noticed that the drug with second highest using frequency, Dixin Eye Drops, is the drug manufactured by the Jinan $2^{\text {nd }}$ hospital itself. The $0.9 \%$ Sodium Chloride Injection is always used as electrolytes for other drugs. It is the duty of the other drugs to play a therapeutic role in many kinds of eye diseases. Mecobalamin Capsules is a cobalamin used in the treatment of peripheral neuropathy, diabetic neuropathy, and as a preliminary treatment for amyotrophic lateral sclerosis. In an eye hospital, it often is used to treat diabetic retinopathy and optic atrophy caused by optic neuritis, diplopia and glaucoma.

Table 1. Top 10 drugs with highest using frequency.

\begin{tabular}{lll}
\hline Drug ID & Drug name & Using frequency \\
\hline 460 & Pranoprofen Eye Drops & 5112 \\
143 & Dixin Eye Drops & 4959 \\
1 & $0.9 \%$ Sodium Chloride Injection & 2249 \\
210 & Compound Tropicamide Eye Drops & 2136 \\
337 & Lubricant Eye Drops & 1782 \\
67 & Sodium Hyaluronate Eye Drops & 1715 \\
300 & Mecobalamin Capsules & 1620 \\
56 & Bendazac Lysine Eye Drops & 1492 \\
463 & Esculin and Digitalisglycosides Eye Drops & 1189 \\
338 & Polyvinyl Alcohol Eye Drops & 1077 \\
\hline
\end{tabular}

Table 2 and table 3 list out the herbal medicines and Chinese patent medicines which are most used respectively.

Table 2. Top 10 herbal medicines with highest using frequency.

\begin{tabular}{|c|c|c|c|}
\hline Drug ID & Drug name in Latin & Drug name in English & Using frequency \\
\hline 131 & Radix Angelicae Sinensis & Chinese Angelica & 280 \\
\hline 243 & Fructus Lycii & Barbary Wolfberry Fruit & 254 \\
\hline 286 & Radix Astragali & Milk Vetch Root & 221 \\
\hline 97 & Paeoniae Radix Rubra & Red Paeony Root & 208 \\
\hline 528 & Radix Rehmanniae & Dried Rehmannia Root & 207 \\
\hline 84 & Rhizoma Atractylodis Macrocephalae Praeparatae & Largehead Atractylodes Rhizome (baked) & 184 \\
\hline 31 & Radix Paeoniae Alba & White Paeony Root & 171 \\
\hline 319 & Flos Lonicerae & Honeysuckle Flower & 154 \\
\hline 166 & Radix Saposhnikoviae & Divaricate Saposhnikovia Root & 149 \\
\hline
\end{tabular}


- Radix Angelicae Sinensis-Chinese Angelica is used as a tonic (blood tonic more specifically) and is heavily used in Chinese Medicine.

- Fructus Lycii-Barbary Wolfberry Fruit is usually used in liver and kidney deficiencies that present with lumbar soreness, sexual dysfunction, dizziness, blurred vision, poor vision, cataract and diabetic symptoms.

- Radix Astragali-Milk Vetch Root invigorates Qi (vital energy) and it also benefits protective Qi and consolidates the exterior of the body.

- Paeoniae Radix Rubra-Red Paeony Root removes heat from the blood, dissipates blood stasis and alleviates pain.

- Radix Rehmanniae-Dried Rehmannia Root clears heat, cools blood, nourishes Yin and promotes body fluids production.

- Rhizoma Atractylodis Macrocephalae Praeparatae-Largehead Atractylodes Rhizome
(baked)-Largehead atractylodes rhizome can invigorate spleen, replenish Qi (vital energy), induce urination, dry the body, control sweating and prevent abortion.

- Radix Paeoniae Alba-White Paeony Root enriches blood, regulates menses, constraints yin, pacifies liver, relieves pain, and arrests sweating.

- Radix Ophiopogonis-Dwarf Lilyturf Tuber nourishes Yin of stomach, lung and heart, clear heat of stomach, lung and heart, calm heart and induce tranquilization.

- Flos Lonicerae- Honeysuckle flower is often used in pus-forming infections, skin sores, pharyngitis, bloody dysentery, cold and flu, and acute infectious diseases.

- Radix Saposhnikoviae-Divaricate Saposhnikovia Root dispels wind and releases exterior, expels wind-damp and alleviates pain.

Because most Chinese patent medicines do not have corresponding name in English, we list the name of Chinese patent medicine in the form of Chinese phonetic.

Table 3. Top 10 Chinese patent medicines with highest using frequency.

\begin{tabular}{lll}
\hline Drug ID & Drug name in Chinese phonetic & Using frequency \\
\hline 464 & Qi Ming Ke Li & 853 \\
214 & Fu Fang Xiong Dan Di Yan Ye & 688 \\
266 & He Xue Ming Mu Pian & 606 \\
219 & Fu Ming Jiao Nang & 571 \\
466 & Qi Ming Jiao Nang & 543 \\
196 & Fu Fang Dan Shen Di Wan & 512 \\
480 & Qing Re San Jie Jiao Nang & 490 \\
249 & Gu Tong Tie Gao & 476 \\
208 & Fu Fang Shi Hu Pian & 334 \\
202 & Fu Fang Jue Ming Pian & 319 \\
\hline
\end{tabular}

- Qi Ming Ke Li is a kind of granule and it is often used for type 2 diabetic retinopathy.

- Fu Fang Xiong Dan Di Yan Ye is a kind of eye drops, whose main constituents are bear bile and borneol. It can be used for the treatment of acute bacterial conjunctivitis and epidemic conjunctivitis.

- He Xue Ming Mu Pian is a kind of tablet in treating eye ground hemorrhage.

- Fu Ming Jiao Nang is a kind of capsule and can be used for the treatment of glaucoma and cataract.

- Qi Ming Jiao Nang is a kind of capsule. It often is used to reinforce liver and kidney, activate blood and improve eyesight.

- Fu Fang Dan Shen Di Wan can promote blood circulation for removing blood stasis, regulate Qi to alleviate pain. It often is used for the treatment of angina pectoris. When eye diseases are considered, it can prevent diabetic retinopathy.

- Qing Re San Jie Jiao Nang is a kind of capsule. It can relieve pain, anti-inflammation. It often is used for the treatment of acute conjunctivitis, acute pharyngitis, acute tonsillitis, acute enteritis and acute dysentery.

- Gu Tong Tie Gao has an effect of removing wind and dispersing cold, clearing meridians and collaterals, eliminating stasis to relieve pain.

- Fu Fang Shi Hu Pian reinforces liver and kidney, regulates Qi and improves eyesight.

- Fu Fang Jue Ming Pian can reinforce liver, replenish Qi. It can be used for the treatment of myopia.

- Drug Use Analysis through Complex Network Theory

In order to analyze the using situation of drugs, four networks of drugs are built. Each node in the network represents a kind of drug. If two kinds of drugs appear in one prescription, an edge is added between the two nodes representing the drugs. Figure 1 is the network of all drugs in the data set. There are 583 nodes and 18464 edges.

Node degree is the number of edges linked with the node [13]. In a social network degree often is used to describe the importance of a node [14]. Network density describes the portion of the potential edges in a network that are actual connections. The average degree of the network is 61.33 and the network density is 0.1045 .

The information about the top 10 drugs whose degree is highest in the network of all drugs, chemical drugs, herbal medicines and Chinese patent medicines are listed in table 4-table 7 respectively. 


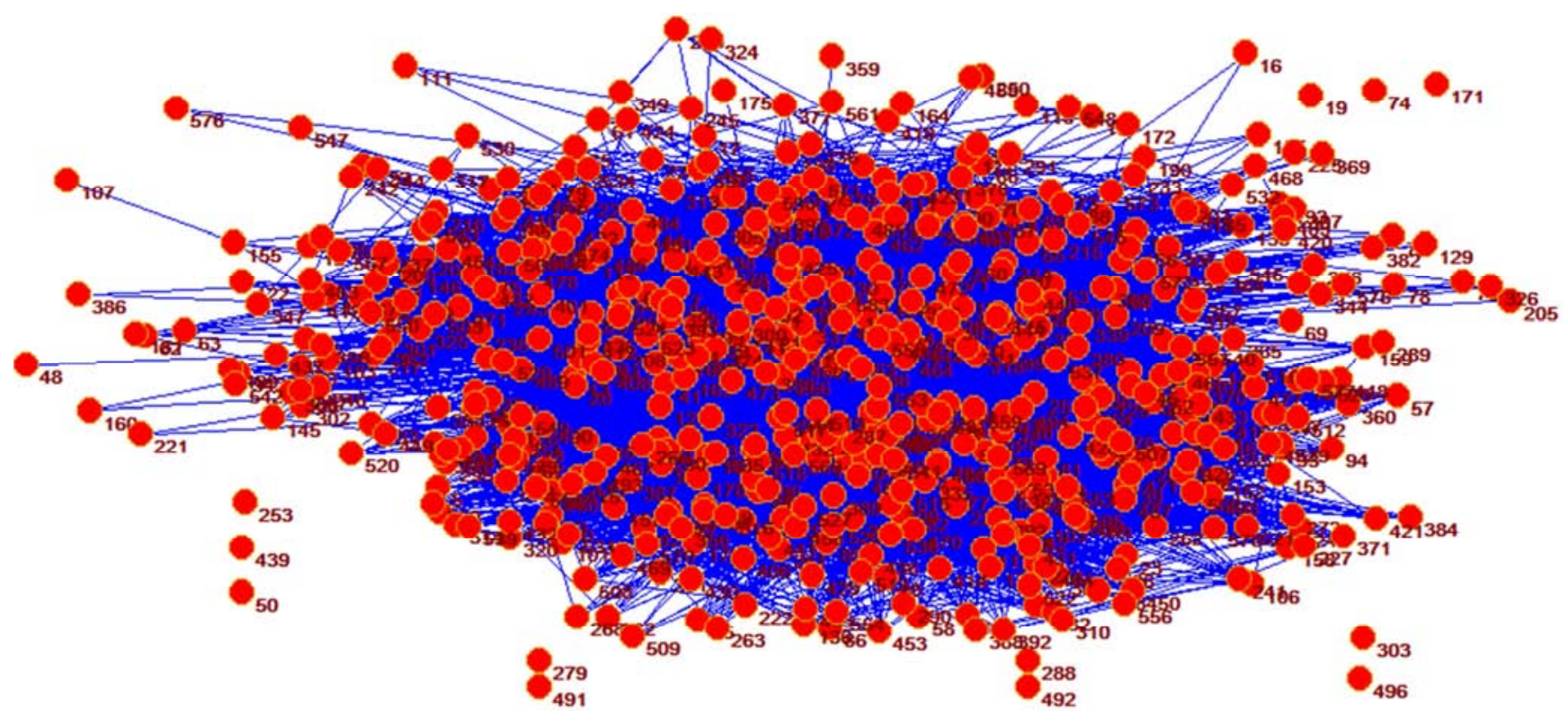

Figure 1. Network of all drugs.

Table 4. Top 10 drugs with highest degree in the network of all drugs.

\begin{tabular}{lll}
\hline Node ID & Drug represented by the node & Node degree \\
\hline 460 & Pranoprofen Eye Drops & 358 \\
143 & Dixin Eye Drops & 352 \\
1 & $0.9 \%$ Sodium Chloride Injection & 266 \\
131 & Radix Angelicae Sinensis & 265 \\
243 & Fructus Lycii & 262 \\
286 & Radix Astragali & 260 \\
413 & Radix Ophiopogonis & 246 \\
11 & Aspirin Enteric-coated Tablets & 236 \\
84 & Rhizoma Atractylodis Macrocephalae Praeparatae & 236 \\
97 & Paeoniae Radix Rubra & 236 \\
\hline
\end{tabular}

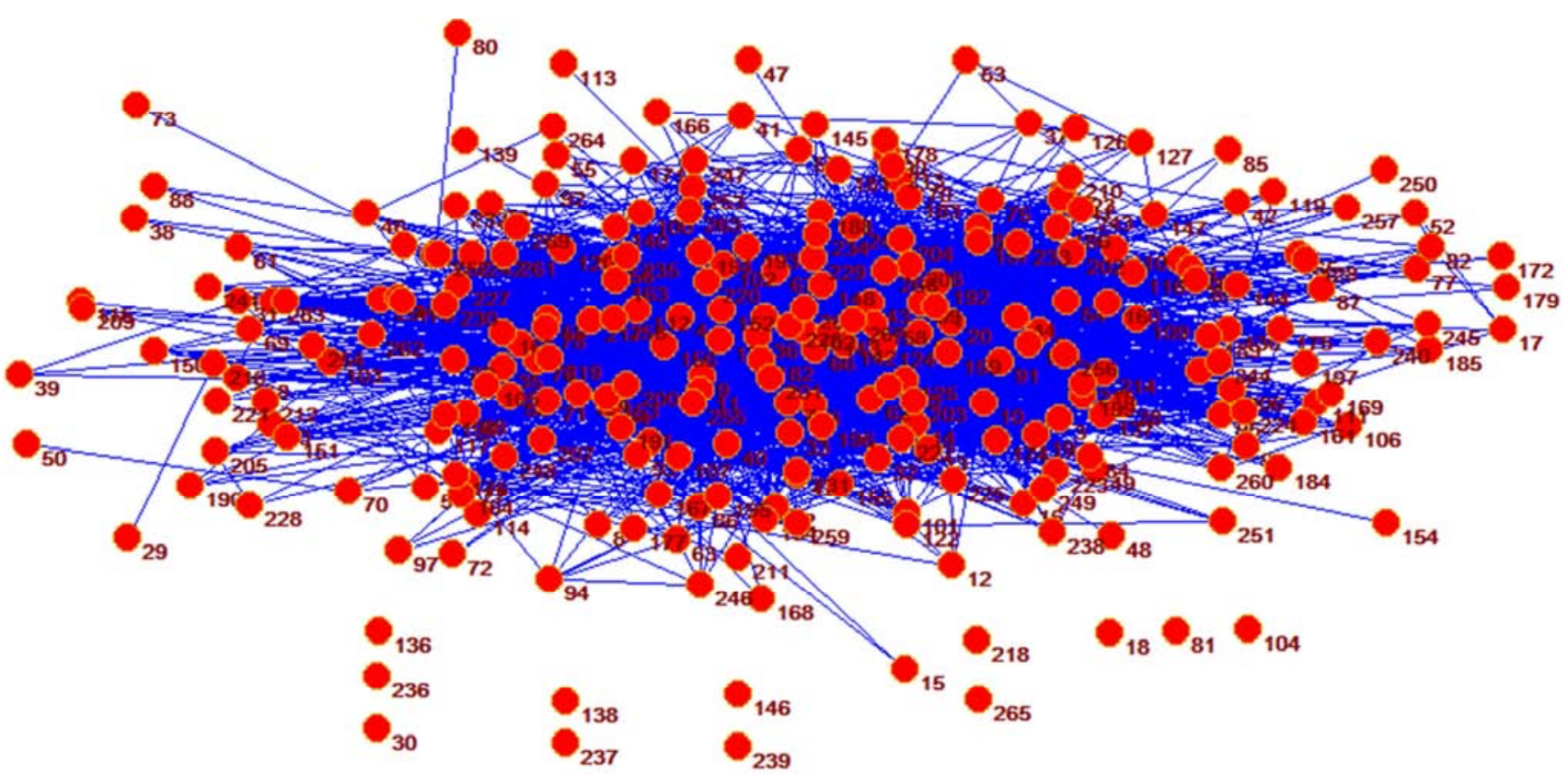

Figure 2. Network of chemical medicines. 
Figure 2 is the network of chemical medicines. It is composed of 269 nodes and 4627 edges. The average degree of the network is 32.39 and the network density is 0.1187 . The information about the top 10 drugs whose degree is highest in the network of chemical medicines is listed out in table 5 .

Table 5. Top 10 drugs with highest degree in the network of chemical medicines.

\begin{tabular}{llll}
\hline Node ID & Drug represented by the node & Drug ID & Node degree \\
\hline 66 & Dixin Eye Drops & 143 & 178 \\
226 & Pranoprofen Eye Drops & 460 & 1 \\
1 & $0.9 \%$ Sodium Chloride Injection & 11 & 166 \\
11 & Aspirin Enteric-coated Tablets & 300 & 133 \\
143 & Mecobalamin Capsules & 210 & 131 \\
108 & Compound Tropicamide Eye Drops & 337 & 129 \\
158 & Lubricant Eye Drops & 56 & 123 \\
36 & Bendazac Lysine Eye Drops & 9 & 122 \\
9 & Alprazolam TABLETS & 402 \\
201 & Losartan Potassium Tablets & 114 \\
\hline
\end{tabular}

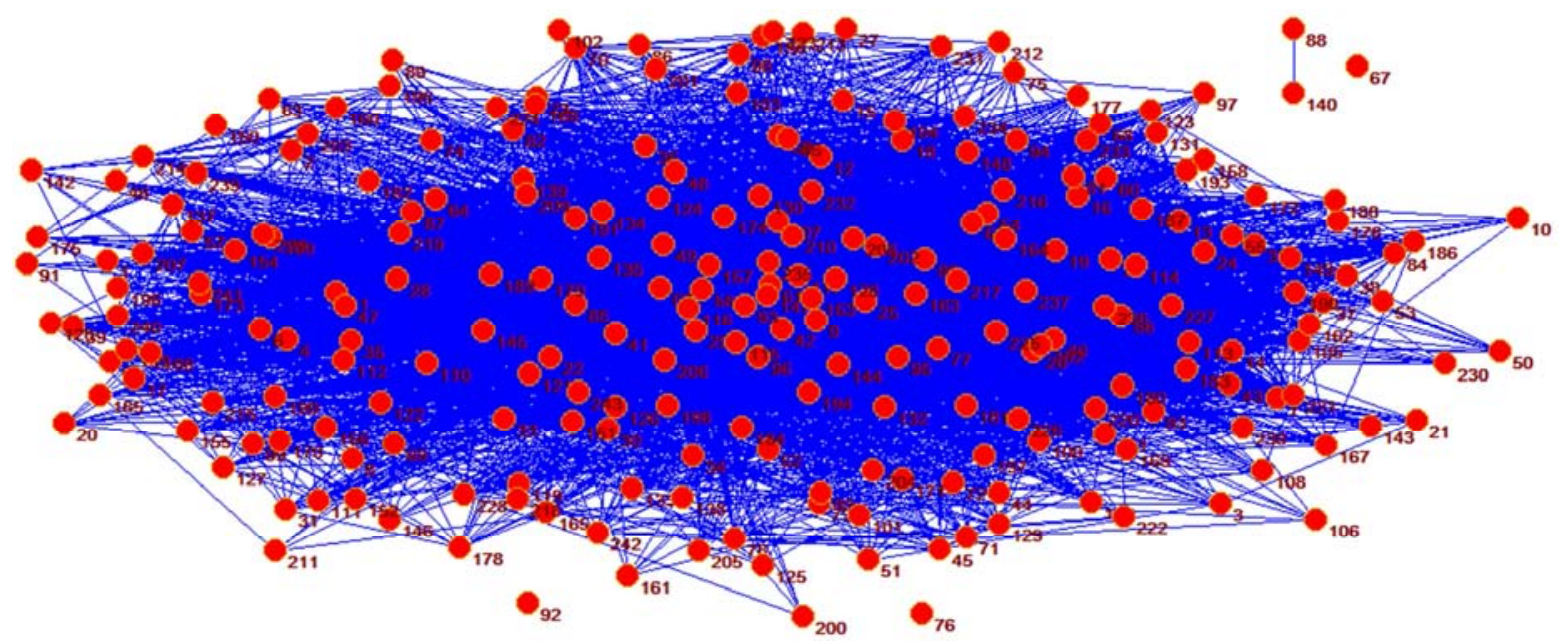

Figure 3. Network of herbal medicines.

Figure 3 is the network of herbal medicines. The network of herbal medicine includes 243 nodes and 6757 edges. The average degree of the network is 53.59 and the network density is 0.2188 . The information about the top 10 drugs whose degree is highest in the network of herbal medicines is listed out in table 6 .

Table 6. Top 10 drugs with highest degree in the network of herbal medicines.

\begin{tabular}{llll}
\hline Node ID & Drug represented by the node & Drug ID & Node degree \\
\hline 116 & Radix Astragali & 286 & 185 \\
61 & Radix Angelicae Sinensis & 131 & 180 \\
93 & Fructus Lycii & 243 & 176 \\
42 & Radix Paeoniae Rubra & 97 & 171 \\
9 & Radix Paeoniae Alba & 31 & 169 \\
157 & Radix Ophiopogonis & 413 & 168 \\
29 & Rhizoma Atractylodis Macrocephalae Praeparatae & 84 & 167 \\
115 & Rhizoma Coptidis & 284 & 159 \\
85 & Radix Glycyrrhizae & 224 & 121 \\
\hline
\end{tabular}

- Radix Paeoniae Alba-White Paeony Root enriches blood, regulates menses, constraints Yin, pacifies liver, relieves pain, and arrests sweating.

- Rhizoma Coptidis-Golden Thread clears heat and dry dampness, purge fire and relieve toxicity.

- Radix Glycyrrhizae-Liquoric Root is often used in conditions like spleen and stomach deficiencies, general weakness, palpitations, breath shortness, productive coughs, spasms in gastric and abdominal regions and limbs, running sores, abscess and poisoning.

- Radix Salviae Miltiorrhizae-Salvia Root activates and nourishes blood, removes blood stasis, regulates menstruation and calms the spirit. 


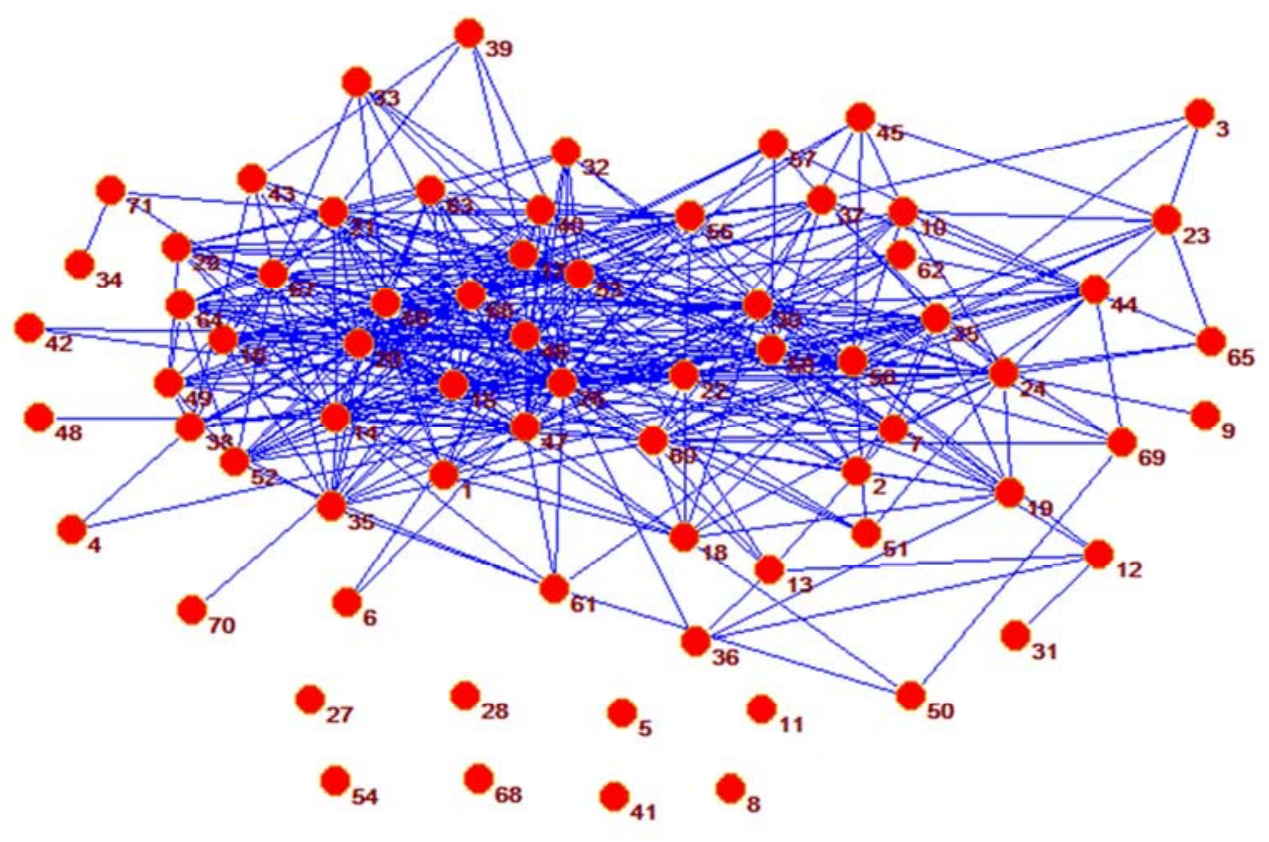

Figure 4. Network of Chinese patent medicines.

Figure 4 is the network of Chinese patent medicines. This network includes 71 nodes and 511 edges. The average degree is 12.34 and the network density is 0.1672 . The information about the top 10 drugs whose degree is highest in the network of Chinese patent medicines is listed out in table 7 .

Table 7. Top 10 drugs with highest degree in the network of Chinese patent medicines.

\begin{tabular}{llll}
\hline Node ID & Drug represented by node & Drug ID & Degree \\
\hline 26 & Gu Tong Tie Gao & 249 & 40 \\
15 & Fu Fang Dan Shen Di Wan & 196 & 30 \\
46 & Ku Gan Ke Li & 353 & 30 \\
53 & Niu Huang Yi Jin Pian & 443 & 30 \\
59 & Qiang Li Pi Ba Lu & 472 & 30 \\
58 & Qi Ming Jiao Nang & 466 & 29 \\
20 & Fu Fang Xi Ling Jie Du Pian & 212 & 28 \\
17 & Fu Fang Gan Cao Kou Fu Rong Ye & 201 & 27 \\
22 & Fu Fang Xiong Dan Di Yan Ye & 214 & 27 \\
30 & He Xue Ming Mu Pian & 266 & 26 \\
\hline
\end{tabular}

\section{Comparison and Conclusions}

From section 3 we can find that the average value of the using frequency for all drugs, chemical drugs, herbal medicines and Chinese patent medicines is 109.91, 178.12, 29.09 and 129.63 respectively. The average using frequency of chemical drugs is highest, then is the Chinese patent medicines, and last is herbal medicines.

Although the using frequency of herbal medicines is lower than chemical drugs and Chinese patent medicines, the relationship among herbal medicines is closer than the relationship among chemical drugs and Chinese patent medicines. This is reflected by the density value of the four networks about the drugs. According to the data from section
4 , the network density of network for chemical drugs, herbal medicines and Chinese patent medicines is $0.1187,0.2188$ and 0.1672 respectively.

The top 3 drugs with highest degree in the network of all drugs are also the three drugs whose using frequency is highest. At the same time, the three drugs whose degree are from fourth to sixth are the top 3 herbal medicines whose using frequency is highest. Seven kinds of drugs in table 1 also are included in table 5. Seven kinds of drugs in table 2 also are included in table 6. The overlap rate of table 3 and table 7 is also up to $50 \%$. Table 1 to table 3 describes the drug's using frequency. Table 4 to table 7 reflects drugs with highest degree. The high overlap rate between these tables shows that node degree of drug network can reflect the drug using frequency well.

\section{Acknowledgement}

This work was supported by science and technology plan general program of Beijing Municipal Education Commission (ID: KM201510037001) and Breeding Project of BWU (Research of Logistics for Low Carbon Economy, NO: GJB20162002).

\section{References}

[1] Gao J, Barzel B, Barabási A L. Universal resilience patterns in complex networks [J]. Nature, 2016, 530 (7590): 307-312.

[2] Pagani G A, Aiello M. Power grid complex network evolutions for the smart grid [J]. Physica A Statistical Mechanics \& Its Applications, 2014, 396 (2): 248-266.

[3] Tian Z, Jia L, Dong H, et al. Analysis of Urban Road Traffic Network Based on Complex Network [J]. Procedia Engineering, 2016, 137: 537-546. 
[4] Yu S Y, Wang H M. Scientific collaboration: a social network analysis based on literature of animal-derived regenerative implantable medical devices: [J]. Regenerative Biomaterials, 2016, 3 (3): 197-203.

[5] Atwi S, Mcmahon D, Scharfman H, et al. The Structure and Function of Complex Netwroks [J]. Neuroscientist A Review Journal Bringing Neurobiology Neurology \& Psychiatry, 2014, 22

[6] Vogler S. Inequalities in medicine use in Central Eastern Europe: an empirical investigation of socioeconomic determinants in eight countries $[\mathrm{J}]$. International Journal for Equity in Health, 2015, 14 (1): 1-19.

[7] Adnan M, Ullah I, Tariq A, et al. Ethnomedicine use in the war affected region of northwest Pakistan [J]. Journal of Ethnobiology and Ethnomedicine, 2014, 10 (1): 125-128.

[8] Huang T P, Liu P H, Lien A S, et al. A nationwide population-based study of traditional Chinese medicine usage in children in Taiwan.[J]. Complementary Therapies in Medicine, 2014, 22 (3): 500-510.

[9] Yen H R, Liang K L, Huang T P, et al. Characteristics of traditional Chinese medicine use for children with allergic rhinitis: a nationwide population-based study [J]. International Journal of Pediatric Otorhinolaryngology, 2015, 79 (4): 591-7.

[10] Huang S K, Ho Y L, Chang Y S. Prescriptions of traditional Chinese medicine, western medicine, and integrated Chinese-Western medicine for allergic rhinitis under the National Health Insurance in Taiwan. [J]. Journal of Ethnopharmacology, 2015, 173: 212-216.

[11] Herbalism, From Wikipedia, the free encyclopedia. https://en.wikipedia.org/ wiki/Herbalism.

[12] Ameh S. Medical Herbalism and Herbal Clinical Research: A Global Perspective [J]. 2011, 1 (4): 99-123.

[13] Aliakbary S, Habibi J, Movaghar A. Feature Extraction from Degree Distribution for Comparison and Analysis of Complex Networks [J]. The Computer Journal, 2015 (9).

[14] Wang W, Tang M, Zhang H F, et al. Epidemic spreading on complex networks with general degree and weight distributions [J]. Physical Review E Statistical Nonlinear \& Soft Matter Physics, 2014, 90 (4): 042803-042803. 